Original Research Paper

\title{
The Participation of Oxidative Stress in Breast Cancer Cells Progression and Treatment Resistance
}

\author{
${ }^{1}$ Poliana Camila Marinello, ${ }^{1}$ Kaliana Larissa Machado, ${ }^{2}$ Rubens Cecchini and \\ ${ }^{1}$ Alessandra Lourenço Cecchini \\ ${ }^{1}$ Laboratory of Molecular Pathology, State University of Londrina, PR, Brazil \\ ${ }^{2}$ Laboratory of Pathophysiology and Free radicals, State University of Londrina, PR, Brazil
}

\author{
Article history \\ Received: 05-02-2014 \\ Revised: 17-02-2014 \\ Accepted: 05-01-2015 \\ Corresponding Author: \\ Alessandra Lourenço Cecchini \\ Laboratory of Molecular \\ Pathology, State University of \\ Londrina, PR, Brazil \\ Email: carolpanis@sercomtel.com.br
}

\begin{abstract}
This article presents a general discussion about the participation of oxidative stress in relevant points related to breast cancer progression in vitro. All of the evidences presented here are based on published papers that used breast cancer cells with different phenotype characteristics in their research. We observed that oxidative stress could modulate by several manners the tumor progression and these effects are directly related to its concentration and time of cell exposure to these substances. Furthermore, oxidative stress produced and released by breast cancer cells is able to interfere in the metabolism of the adjacent normal cells in a manner that improve the survival of the neoplastic cells. In relation to breast cancer treatment, the role of oxidative stress is complex. At the same time that it can be responsible to the induction of cell death, oxidative stress can also modulate pathway that leads to increased expression of anti-apoptotic and resistance-related proteins. Therefore, the participation of oxidative stress in breast cancer is complex and very broad and its better understanding could be important to the development of more effective therapeutic strategies for the different forms clinically found of the disease.
\end{abstract}

Keywords: Breast Cancer, Oxidative Stress, Chemoresistance, Cell Culture

\section{Introduction}

The oxidative stress is caused by an imbalance between pro-oxidant substances and intracellular antioxidant defense system promoting the establishment of an environment composed of highly unstable molecules that are able to react with significant cellular structures such as proteins, lipids and DNA. Despite its involvement in carcinogenesis, Reactive Oxygen Species (ROS) not only exhibit adverse health effects because it participates of different intracellular signaling pathways, such as activation of signal transduction cascades that lead to the control of cell proliferation and differentiation, maturation and apoptosis induction (Gago-Dominguez et al., 2007).

The breast cancer represents a public health problem worldwide showing increasing rates of incidence and mortality each year. By presenting high heterogeneity due to many phenotypes and different clinical and morphological presentations, the disease has a wide variation in clinical responses, which hinders the cancer treatment availability. Within this context, the term targeted therapy has been used to describe new drugs that were planned based on the knowledge of the fundamental molecular pathology of the disease.

The target therapy have been created because of the existence of different characteristics in the tumor cell lines, so the mechanisms by which the hyper proliferation occurs in these cells varies according to their characteristics. For example, estrogen and progesterone have a proliferation-inducing effect and are involved in the carcinogenesis of breast cancer and different drugs are currently available to decrease its effects. Furthermore, studies have shown that more than $30 \%$ of mammary carcinomas over express particularly the HER 2 receptor and this increased expression is most frequent in cells that do not possess Estrogen Receptors (ER) than in those ones that do. This cancer type has characteristics that are more aggressive and the target of its therapy consists of the administration of a monoclonal antibody specific for HER 2 or the administration of inhibitors of tyrosine kinases EGFR/HER2, thereby blocking the intracellular signaling.

There are also tumor cells that do not express hormone receptors (for estrogen and progesterone) neither HER 2, they are called triple-negative strains 
displaying poor prognosis and high rates of recurrence with no specific treatment, in this case, systemic chemotherapy is the only option available (Howe and Brown, 2011). However, in despite of the advances in the therapy of this disease in recent years, there is no treatment to ensure satisfactory response in different types of breast cancer, yet. For this reason, many studies have been conducted through culture and maintenance of tumor cell lines in vitro to improve the understanding of the mechanisms related to tumor progression in an attempt to develop new therapeutic strategies or even to optimize the existing treatments.

There is no consensus about the harmful or beneficial role of ROS in the carcinogenesis and progression of breast cancer. However, the knowledge of its importance in the different mechanisms related either to carcinogenesis and tumor progression or with factors related to the disease cure is necessary. This understanding could be useful in the prevention and in the development of new therapies to improve patients quality of life. Therefore, the purpose of this paper is to review the information available in the literature about the oxidative stress participation in tumor progression, in tumor microenvironment influence and in the treatment response.

\section{Methodology}

This study was performed through a literature review searching information's about oxidative stress in breast cancer cells. The research was conducted using the electronic PubMed search engines and "oxidative stress and breast cancer cells" was used as key word. The search found 776 results, including original articles and reviews. After the research, it was concluded that the importance of the involvement of the oxidative stress in breast cancer cells can be divided into three fundamental points: Oxidative stress and tumor progression; oxidative stress and tumor microenvironment influence and oxidative stress and treatment response. The articles used in this review were published between 2001 and 2013 and were selected in accordance with their relevance in the subject studied.

\section{Oxidative Stress and Tumor Progression}

Changes in the intracellular redox state are able to interfere with the activation of several transcription factors that are important in the control of cell proliferation and DNA repair, such as p53 and the Nuclear transcription Factor kB (NF-kB) (Lu and Holmgren, 2012). Furthermore, ROS also participate in different modulators intracellular pathways, such as the Thioredoxin antioxidant system (Trx). In its reduced state, Trx binds to regulatory kinase apoptosis signal 1 (ASK1) and inhibits its ability to induce apoptosis. When this protein is oxidized, it dissociates from ASK1, stimulating apoptosis.
Many antioxidants are able to prevent tumor formation induced by carcinogens and also to inhibit the transformation of cells in the presence of carcinogens, in vitro. The mechanisms by which the antioxidants attenuate the toxic effects of xenobiotics and oxidative stressors are still not fully understood. It is known that due to their electrophilic nature, antioxidants are able to destabilize the cytoplasmic complex Keapl-Nrf2 allowing the nuclear transcription factor associated with E2 (Nrf2) accumulation in the nucleus, associating to other factors and linking into the Antioxidant Response Element (AREs) in the genes regulatory regions encoding enzymes and carriers that protect cells from oxidative stress and xenobiotics. AREs examples are glutathione peroxidase, glutathione S-transferase, Hemeoxigenase-1 (HO-1) and NADPH Quinone Oxidoreductase 1 (NQO1), which regulate metabolism and the intracellular levels of the antioxidant peptide, glutathione. Similarly, genes encoding proteins capable of expelling chemotherapeutic agents out of the cell, often found in breast cancer cells resistant to chemotherapy, also have AREs in its promoter region, showing that the redox imbalance also influences the chemoresistance in tumor cells and both antioxidants and chemotherapeutic agents are able to induce gene expression mediated by AREs. It has been reported that tumor cell lines treatment with antioxidants can inhibit the activity of factors that contribute to the transformed cell phenotype, including the Signal Transducer and Activation of Transcription 3 (STAT 3), Phosphatidylinositol 3-Kinase (PI3K) and Extracellular protein Kinase regulated by signal (ERK 1/2). It demonstrates that the addition of antioxidants at nontoxic concentrations to the chemotherapeutic regimens could increase treatment effectiveness (De Larco et al., 2010).

The oxidative stress role in tumor cells seems to be dependent on its intensity and duration. While high concentration promote apoptosis induction, sub-lethal concentration are able to stimulate cell proliferation in vitro by, among all, the activation of Mitogen-Activated Protein Kinases pathway (MAPK) and the prolonged exposure to these concentrations induced apoptosis resistance through the induction of antioxidants molecules and the production of antiapoptotic proteins. The free radicals also stimulate the tumor cell migration through the activation of pathways that regulate the actin cytoskeleton dynamics in the cell, such as MAPK and also by inducing metalloproteinases production that are capable of degrading extracellular matrix (Brown and Bicknell, 2001).

Another important factor for tumor progression is the rate of intracellular autophagy. In mammalian cells, an important mechanism in autophagy regulation is the complex 1 of the mammalian Target of Rapamycin 
(mTORC1), which act as a sensor of intracellular nutrients availability. When there is an appropriate concentration of intracellular nutrients, this complex is activated, inhibiting autophagy by inactivating essential components for the nucleation of autofagossoma. In situations of nutrients depletion or mTORC1 pharmacological inhibition, the autophagy is stimulated by several mechanisms, such as the activation of the kinase activated by AMP, the AMPK. The autophagy role in tumorigenesis is complex and appears to be dependent of the cell context. The malignant neoplastic cells metabolism is characterized by the increase of speed, but the decrease in the efficiency of the energy production. This metabolic profile is known as the Warburg effect and mutations in some ontogenesis are responsible for the increase in this effect, with higher uptake of glucose and increased transcription of enzymes involved in its metabolism. These mutations are also able to inhibit autophagy, by mTORC1 activation, supporting the hypothesis that the catabolic processes were suppressed in tumor cells, thus facilitating cell mass accumulation.

However, new evidences obtained through the analysis of mutations in other oncogenes such as K-Ras, for example, demonstrates that autophagy is required during the tumor transformation process and deletions in tumor suppressors genes, such as p53, lead to activation of the autophagy. Within this context, the mitochondria also has an important role. Recent studies have shown that mitochondrial activity is important for tumor progression, since the ammonia produced in the process of glutamine utilization in the tricarboxylic acid cycle is responsible for increase the basal autophagy, protect cells from apoptosis and limit proliferation in conditions of metabolic stress. Autophagy is also important when the nutrients supply is decreased such as in hypoxia. In this case, the survival-mediated autophagy is essential for tumor growth and tumor progression since hypoxia induces oxidative stress with greater ROS production that interact with the mitochondria, damaging it. The mitochondrial damage induces autophagy for the elimination of the damaged organelle (Fig. 1), which is very important because if this removal did not occur mitochondrial injury could be responsible for cellular apoptosis activation (Lozy and Karantza, 2012).

One of the main carcinogenic mechanisms related to estrogen includes its oxidative metabolism with subsequent ROS formation. In MCF-7 (human breast adenocarcinoma, hormone receptor-positive cells), physiological estrogen concentrations are able to induce oxidative stress, particularly from the mitochondrial origin. Furthermore, the 8-Hydroxydeoxyguanosine (8$\mathrm{OHdG}$ ) levels, a marker of oxidative DNA damage, in MCF-7 is nine times greater than the levels in triple negative cells MDA-MB-231, which do not have hormone receptor. The expression inhibition of the Estrogen Receptor type alpha $(\mathrm{ER} \alpha)$ in MCF-7 significantly reduces the induction of $8-\mathrm{OHdG}$ formation by the hormone in the cells, suggesting that ROS play a crucial role in the pathogenesis of this type of breast cancer (Karihtala et al., 2011). In breast cancer cells that have HER amplification, there is not much information about the ROS role in tumorigenesis but it is known that this receptor amplification, especially HER2, is associated with a worse prognosis, a more aggressive cellular phenotype and increased capacity for metastasis. The intracellular signaling pathways upregulated by HER overexpression is the PI3K and the cellular homologous Protein to the oncogene vAkt (PI3K/Akt), the activation of this pathway is responsible for the increased cell proliferation, contributing for tumorigenesis. HER2 overexpression increases the number of tumor cells initiators, called Tumor Stem Cells (TSC), both in normal breast cells as in malignant cells. These TSC, in addition to being responsible for carcinogenesis, also contribute to treatment resistance and the induction of these cells by HER 2 amplification is the main mechanism by which this receptor induces tumorigenesis and the invasive capacity of these cells (Korkaya et al., 2008).

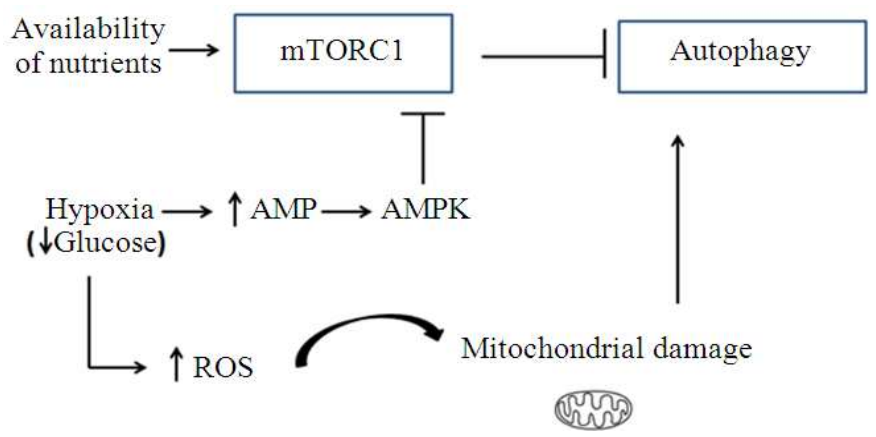

Fig. 1. Regulatory mechanisms of autophagy in response to changes in nutrients supply. When the cell has an adequate supply of nutrients, the mTORC1pathwayis activated and autophagy is inhibited. When the availability of nutrients is lower as in hypoxia, for example, the AMP concentration increases and AMP kinase is activated, consequently inhibiting mTORC1 and inducing autophagy. Hypoxia leads to increased generation of reactive oxygen species that can cause mitochondrial damage and induce autophagy 
The cell lines that do not have receptors for estrogen and progesterone and HER2 amplification are called triple-negative. These cells have markers that are normally expressed in basal and myoepithelial normal breast cells and neoplastic transformation of these cells is often associated with mutation or BRCA1 gene deletion, required for the repair of damaged DNA. The depletion of the BRCA1 messenger RNA in breast cells leads to decreased differentiation and increased cell proliferation, therefore, the mutation in this gene leads to the accumulation of genetically unstable cells with high proliferative capacity, which can eventually can become tumor cells (Bouwman and Jonkers, 2008). This cell type is characterized by one of the most aggressive phenotypes among breast cancer cells and there is evidence that have greater resistance to cell death induced by oxidative stress, possibly due to the higher concentration of protector molecules in these cells. For example, triple-negative cells have increased expression from Proteic Tyrosine Phosphatase Non-receptor type 12 (PTPN12) protecting the cell from the abnormal ROS accumulation and oxidative stress-induced cell death (Harris et al., 2013). Moreover, experiments using the cell line HCC 1937, which has mutation in BRCA1 showed that co-culture with HCC 1937 and fibroblasts induces oxidative stress in the tumor microenvironment and this induction is essential for the survival of tumor cells, since treatment with antioxidants induces apoptosis in these cells. The oxidative stress inducing effect is inhibited by complementation with wild gene BRCA1, showing that the induction of stress occurs due to gene deficiency. Moreover, the co-culture also leads to fibroblasts increased expression of the protein responsible for the lactate release out of the cells, the MCT4, which serves as an energy source for tumor cells, promoting their proliferation (MartinezOutschoorn et al., 2011).

\section{The Influence of the Tumor Microenvironment}

Recently, the relationship between cancer cells and the tumor microenvironment, formed by the adjacent stroma, has been studied and appears to interfere with different mechanisms of tumorigenesis, ranging from initiation and development of metastases as in treatment sensitivity. An important example of these interactions between cells and the adjacent stroma is the metabolic coupling that consists in an inversion of the Warburg effect, in which tumor cells use the nutrients provided by stromal fibroblasts as substrate to keep their metabolism, which becomes predominantly mitochondrial. This happens because the high ROS levels produced in tumor cells due to their elevated metabolism affect the adjacent fibroblasts and cause oxidative stress in these cells with mitochondrial injury and subsequent induction of organelle autophagy. It promotes increased glycolysis with release of nutrients such as lactate and ketones, which will be used as substrates in the neoplastic cells oxidative phosphorylation. Moreover, the ROS generation by fibroblasts also cause DNA damage and genomic instability in cancer cells, promoting a more aggressive tumor phenotype (Fig. 2) (Lozy and Karantza, 2012). The fibroblasts in the stroma also increases the microenvironment vascularization. When exposed to oxidative stress conditions, these cells are activated and change into my fibroblasts, more mobile and contractile and with the ability to secrete high levels of growth factors, cytokines and metalloproteinases. It will help in the proliferation and in the invasiveness of tumor cells, besides producing hydrogen peroxide which will lead to more fibroblasts activation and also cause tumorigenics changes in epithelial cells (Jesierska-Drutel et al., 2013).

The interaction with the tumor microenvironment may also affect the sensibility to antineoplastic treatment, as is the case of decreased sensibility to tamoxifen, which can be induced by the cells that constitute the tumor microenvironment, since the coculture of MCF-7 and stroma fibroblasts decreased apoptosis rates in response to treatment with the drug when compared to cells cultured alone. The resistance occurs due to the induction by tamoxifen, in co-culture with fibroblasts, of the TIGAR over expression, a gene regulated by $\mathrm{p} 53$ that simultaneously inhibits glycolysis, autophagy, apoptosis and decreased ROS generation, thus promoting the mitochondrial oxidative metabolism and protecting cancer cell from apoptosis induced by tamoxifen. This suggests that the increase in mitochondrial function is related to resistance to this drug, which is proven through the use of substances that decrease the mitochondrial activity with subsequent recovery of cell sensibility to the medicament as discussed earlier, tumor progression in breast cancer can occur for different ways due to the cells heterogeneity, as illustrated in Fig. 3. In addition, co-culture of MCF-7 with fibroblast also induces resistance of this cell line to other drugs such as doxorubicin and the veliparib, also known as ABT-888, which the mechanism of action is completely different of tamoxifen, indicating that the tumor microenvironment plays an important role in the general mechanism of drug resistance induction (Martinez-Outschoorn et al., 2011). 


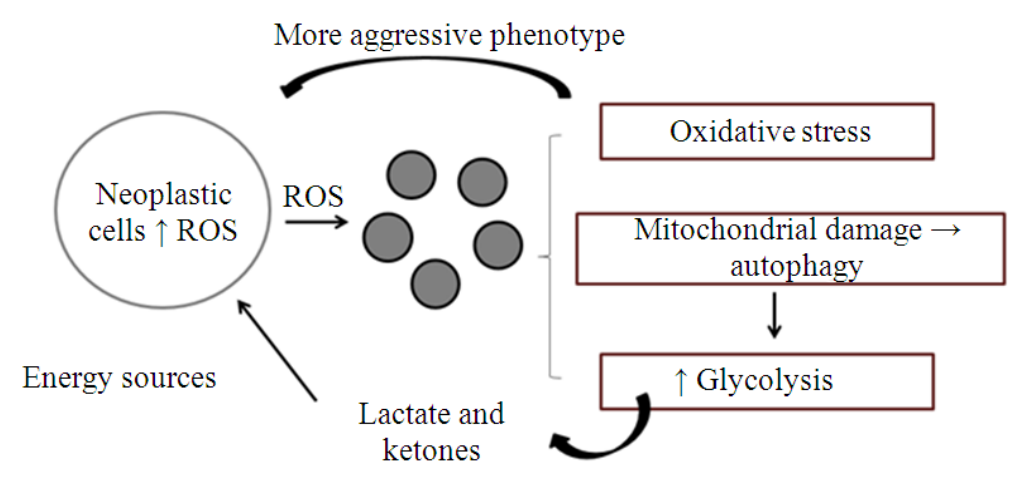

Stroma fibroblasts

Fig. 2. The in fluence of the tumor micro environment in a more aggressive phenotype of cancer cells. The neoplastic cells have high amounts of ROS due to its accelerated metabolism. These ROS can attack the stroma fibroblasts, causing mitochondrial damage and the induction of organelle autophagy, which leads to increased glycolysis, to compensate the energy production, increasing the production and release of lactate and ketone by these cells, to be used by tumor cells as a source of energy. The increase in the fibroblasts oxidative stress will also reach the neoplastic cells causing DNA damage, generating genomic instability, which contributes to a more aggressive tumor phenotype

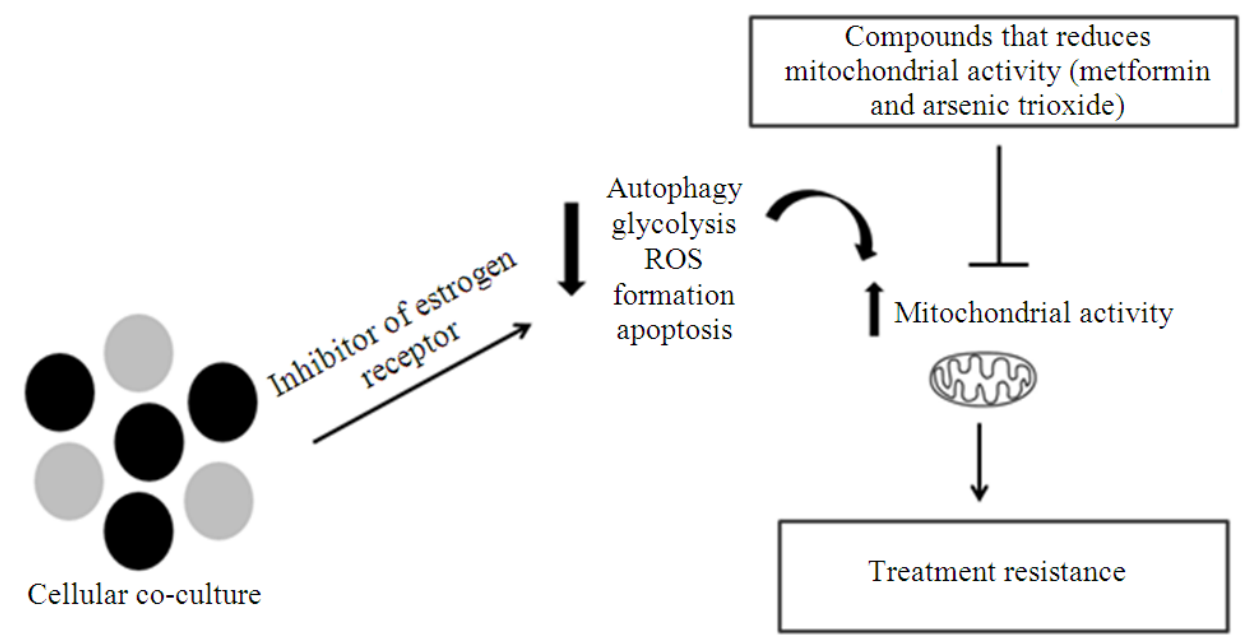

Cells with estrogen receptors

Cancer associates fibroblasts

Fig. 3. Mechanisms of resistance induction to anti-estrogen therapy by cells of the tumor microenvironment. Tamoxifen treatment (estrogen-receptor inhibitor) in co-culture of cells that possess hormone receptor and cancer associated fibroblasts induces the over expression of TIGAR gene, responsible to inhibiting glycolysis, autophagy, apoptosis and ROS generation, promoting mitochondrial oxidative metabolism and protecting cancer cell to the tamoxifen-induced apoptosis. The mechanism of resistance induction is related to mitochondria since the use of substances that reduce its activity render the cells sensitive to treatment again

\section{Oxidative Stress Participation in Treatment Response}

As discussed earlier, tumor progression in breast cancer can occur for different ways due to the cells heterogeneity that leads to carcinoma and so many types of drugs are used taking into account these differences, called targeted therapies. However, the availability of more specific drugs do not exclude the use of cytotoxic chemotherapy, which is still one of the most widely used, especially in the advanced stages the disease. Among the several available chemotherapeutic agents, many exerts their apoptosis inductive effect in tumor cells due to increased ROS formation such as 
superoxide anion radical $\left(\mathrm{O} 2^{-\bullet}\right)$, Hydrogen peroxide $\left(\mathrm{H}_{2} \mathrm{O}_{2}\right)$ and hydroxyl radicals $\left(\mathrm{OH}^{\bullet}\right)$, as is the case of doxorubicin, cyclophosphamide, cisplatin and paclitaxel, for example (Glorieux et al., 2011). Furthermore, oxidative stress is frequently involved in the drugsmechanisms of action that have been studied in the breast cancer treatment, both when used alone or in combination with other agents.

The association of a fucose rich polysaccharide extract with tamoxifen, cisplatin and paclitaxel in MCF7 cells (hormone receptors) and MDA-MB-231 (triple negative) significantly enhanced the induction of cell death by these drugs. It was mediated by regulating the expression of proteins of the Bcl-2 family, the activation of ERK1/2 and Akt signaling pathways and through the control of the reactive oxygen species production within the cell. Tamoxifen is a selective antagonist of estrogen receptor and is widely used both in early as in more advanced stages of hormone dependent breast cancer and its action is based on the proliferation reduction and apoptosis induction. The mechanisms related to apoptosis induction by the drug include increased oxidative stress, induction of the expression of modulators proteins and the activation of important pathways such as ERK1/2, the Transforming Growth Factor $\beta$ (TGF- $\beta$ ), Protein Kinase $\mathrm{C}$ (PKC) and members of the family of $\mathrm{Bcl}-2$ proteins. The Paclitaxel is an antimicrotubule drug that acts on cancer cells through inducing apoptosis and cell cycle arrest and its effects are also related to changes in signaling pathways and gene expression, as activation of MAPK, Raf-1 (viral oncogene homolog 1 murine leukemia vraf-1), tyrosine kinase protein and Bcl-2 family proteins like $\mathrm{Bcl}-2$, Bad and Bcl-xL. The cisplatin has its cytotoxic effect through interference with mechanisms of transcription and DNA replication leading to activation of cell cycle regulators and the arrest of cell cycle at G2. Apoptosis induction by this drug occurs through the different pathways such as signaling by death receptor, MAPK, Akt, p53 and activation of mitochondrial pathways (Zhang et al., 2013).

One of the main problems in the therapy of breast cancer is treatment resistance, as much of the agents used interfere with the intracellular ROS levels is to be expected that changes in pathways that regulate the redox state participate in the intracellular mechanisms that lead to treatment resistance. The development of Multiple Drug Resistance (MDR) after an initial therapy response limits the success of treatment in this disease. Multi-drug resistant cells can be selected in vitro by treatment with increasing concentrations of chemotherapeutic and resistance induction occurs simultaneously with several biochemical changes. In MCF-7 cells treated with doxorubicin, the multidrug resistance is followed by decreased intracellular drug concentration due to P-glycoprotein (PgP) overexpression and Multidrug Resistance Related Protein (MRP1), increased activity of glutathionedependent antioxidant enzymes, changes in the main drugs target, the topoisomerase II and increased DNA repair. In this cell line, the use of substances capable of increase the intracellular oxidative stress as Tempol enhances the doxorubicin effect on both sensitive and resistant cells (Gariboldi et al., 2006). Similarly, in triple negative cells, MDA-MB-231, the use of a $\omega-3$ polyunsaturated fatty acid, able to increase the levels of the product of lipid oxidation within the cell, the malondialdehyde, potentialized the doxorubicin action in these cells (Mahéo et al., 2005). Moreover, oxidative stress induces the activation of Calcium-dependent Kinase/calmodulin (CaMKs) and these kinases are responsible for upregulation of anti-apoptotic signaling pathways such as Akt, ERK and NF-kB and the use of inhibitors of these kinases in MCF-7 cells decreased doxorubicin resistance. For this reason CaMKs inhibitors have been investigated due to its important participation in the in the fight against resistance to drugs that act by increasing the intracellular oxidative stress (Rodriguez-Mora et al., 2006).

The oxidative stress also has different actions in resistance to anti-estrogen therapy in hormone-dependent breast cancer. Some of the drugs used, such as tamoxifen, an ER inhibitor, are able to increase the production of cellular ROS and, at the same time that this effect assists in the tumor growth restraint, by decreasing mitochondrial function and impairing cell proliferation, it can lead to increased expression of redox sensitive transcription factor AP-1 that could induce drug resistance. Another mechanism associated with anti-estrogen therapy resistance is the presence of large amounts of neoregulina growth factor. This soluble growth factor is able to bind to HER2, HER3 and HER4 receptors, inducing homo or heterodimerization and activating several signaling pathways that culminate in a stimulatory effect on the breast cancer cells growth and this effect is important on the cell types that have hormone receptors and concomitant HER amplification. While the activation of the signaling of neoregulina axis be considered a bad prognostic factor in breast cancer and induce treatment resistance to HER inhibitors and hormonal therapy, the expression of this growth factor may have a beneficial effect when the disease is treated with ligands of the Peroxisome Proliferator-Activated gamma Receptors (PPAR $\gamma$ ), as troglitazone. The PPAR $\gamma$ receptors are a subtype of peroxisome proliferatoractivated receptor, PPAR belonging to the family of nuclear hormone receptors and are expressed in different types of human cancers playing an important role in proliferation, differentiation and apoptosis in cancer cells. In particular, PPAR activation by its ligands is able to 
reduce growth through the induction of cell differentiation or apoptosis. In human breast carcinoma cells, SKBr3 (HER2 amplified), MCF-7 (ER ${ }^{+} \mathrm{PR}^{+}$) and MDA-MB-453 (triple negative), the combination of neoregulina and troglitazone presented a synergistic effect, inducing cell death. This effect was mediated by oxidative stress, with an increase in generation of mitochondrial superoxide anion and disruption of mitochondrial potential. The pretreatment with antioxidantsattenuate the effect produced by the combined treatment, indicating that this combination can serve as a basis for the development of a new strategy in the different types of breast cancer treatment (Park et al., 2011).

\section{Conclusion}

The role of oxidative stress in breast cancer is complex and divergent and seems to be related to its intensity and time of cellular exposition. The studies using breast cancer cell lines with different phenotype characteristics showed that, in most cases, the increase in oxidative stress is related to cell death. However, frequently more aggressive cell lines show resistance to the oxidative stress induced cell death. This resistance can be due to the higher concentration of antioxidant substances, but these resistant cells, with high level of oxidative stress, are able to interfere with the metabolism of adjacent normal cells in a manner that promotes their survival.

Oxidative stress also appears to play an important role in the mechanism of resistance to anticancer treatment, since it has been demonstrated its ability to activate pathways responsible for the up regulation of anti-apoptotic mechanisms. Furthermore, genes encoding proteins capable of expelling chemotherapeutic agents out of the cell, often found in chemoresistant cells, have AREs in its promoter region, being able to stimulate increased production of intracellular antioxidants. Therefore, the participation of the of oxidative stress in different important pathways related to proliferation and treatment resistance of breast cancer cells needs to be better investigated and it could be important to the development of more effective therapeutic strategies for the different forms clinically found of the disease.

\section{Author's Contributions}

All authors equally contributed in this work.

\section{Ethics}

This article is original and contains unpublished material. The corresponding author confirms that all of the other authors have read and approved the manuscript and no ethical issues involved.

\section{References}

Bouwman, P. and J. Jonkers, 2008. Mouse models for BRCA1 associated tumorigenesis. Cell Cycle, 7: 2647-2653. DOI: 10.4161/cc.7.17.6266.

Brown, N.S. and R. Bicknell, 2001. Hypoxia and oxidative stress in breast cancer Oxidative stress: Its effects on the growth, metastatic potential and response to therapy of breast cancer. Breast Cancer Res., 3: 323-327. DOI: 10.1186/bcr315

De Larco, J.E., C.A. Park, H. Dronava and L.T. Furcht, 2010. Paradoxical roles for antioxidants in tumor prevention and eradication. Cancer Biol. Therapy, 9: 362-370. DOI: 10.4161/cbt.9.5.10895

Gago-Dominguez, M., X. Jiang and J.E. Castelao, 2007. Lipid peroxidation, oxidative stress genes and dietary factors in breast cancer protection: A hypothesis. Breast Cancer Res., 9: 201-211. DOI: 10.1186/bcr1628

Gariboldi, M.B., F. Terni, R. Ravizza, S. Meschini and M. Marra et al., 2006. The nitroxide Tempolmodulates anthracycline resistance in breast cancer cells. Free Radical Biol. Medicine, 40: 1409-1418.

DOI: 10.1016/j.freeradbiomed.2005.12.011

Glorieux, C., N. Dejeans, B. Sid, R. Beck and P.B. Calderon et al., 2011. Catalase overexpression in mammary cancer cells leads to a less aggressive phenotype and an altered response to chemotherapy. Biochemical Pharmacol., 82: 1384-1390. DOI: 10.1016/j.bcp.2011.06.007.

Harris, I.S., H. Blaser, J. Moreno, A.E. Treloar and C. Gorrini et al., 2013. PTPN12 promotes resistance to oxidative stress and supports tumorigenesis by regulating FOXO signaling. Oncogene.

DOI: $10.1038 /$ onc.2013.24

Howe, L.R. and P.H. Brown, 2011. Targeting the HER/EGFR/ErbB family to prevent breast cancer. Cancer Prev Res., 4: 1149-1157. DOI: 10.1158/1940-6207.CAPR-11-0334

Jesierska-Drutel, A., S.A. Rosenweig, adn C.A. Neumann, 2013. Role of oxidative stress and the microenvironment in breast cancer development and progression. Adv. Cancer Res., 119: 107-125. DOI: 10.1016/B978-0-12-407190-2.00003-4

Karihtala, P., S. Kauppila, Y. Soini and Arja-JukkolaVuorinen, 2011. Oxidative stress and counteracting mechanisms in hormone receptor positive, triplenegative and basal-like breast carcinomas. BMC Cancer, 11: 262-268. DOI: 10.1186/1471-2407-11-262

Korkaya, H., A. Paulson, F. Iovino and M.S. Wicha, 2008. HER2 regulates the mammary stem/progenitor cell population driving tumorigenesis and invasion. Oncogene, 27: 61206130. DOI: $10.1038 /$ onc. 2008.207 
Lozy, F. and V. Karantza, 2012. Autophagy and cancer cell metabolism. Semin Cell Dev. Biol., 23: 395401. DOI: $10.1016 /$ j.semcdb.2012.01.005

Lu, J. and A. Holmgren, 2012. Thioredoxin system in cell death progression. Antioxid Redox Signal, 17: 1738-1747. DOI: 10.1089 /ars.2012.4650

Mahéo, K., S. Vibet, J.P. Steghens, C. Dartigeas and M. Lehman et al., 2005. Differential sensitization of cancer cells to doxorubicin by DHA: A role for lipoperoxidation. Free Radical Biol. Med., 39: 742751. DOI: 10.1016/j.freeradbiomed.2005.04.023

Martinez-Outschoorn, U.E., A. Goldberg, Z. Lin, Y. Ko and N. Flomenberg et al., 2011. Anti-estrogen resistance in breast cancer is induced by the tumor microenvironment and can be overcome by inhibiting mitochondrial function in epithelial cancer cells. Cancer Biol. Therapy, 12: 924-938. DOI: $10.4161 / \mathrm{cbt} \cdot 12.10 .17780$
Park, B., S. Lee, D.B. Stolz, Y.J. Lee and B. Lee, 2011. Synergistic Interactions between Heregulin and Peroxisome Proliferator-activated Receptor- $\gamma$ (PPAR $\gamma$ ) agonist in breast cancer cells. J. Biol. Chem., 286: 20087-20099. DOI: 10.1074/jbc.M110.191718

Rodriguez-Mora, O.G., M.M. Lahair, M.J. Evans, C.J. Kovacs and R.R. Allison et al., 2006. Inhibition of the CaM-kinases augments cell death in response to oxygen radicals and oxygen radical inducing cancer therapies in MCF-7 human breast cancer cells. Cancer Biol. Therapy, 5: 1022-1030. DOI: $10.4161 / \mathrm{cbt}$.5.8.2910

Zhang, Z., K. Teruya, T. Yoshida, H. Eto and S. Shirahata, 2013. Fucoidan extract enhances the anticancer activity of chemotherapeutic agents in MDAMB-231 and MCF-7 breast cancer cells. Mar. Drugs, 11: 81-98. DOI: 10.3390/md11010081 